\title{
Shape Based Identification of Proteins in Volume Images
}

\author{
Ida-Maria Sintorn and Gunilla Borgefors \\ Centre for Image Analysis, \\ Swedish University of Agricultural Sciences, \\ Lägerhyddsvägen 3, SE-75237 Uppsala, Sweden \\ \{ida, gunilla\}@cb.uu.se
}

\begin{abstract}
A template based matching method, adopted to the application of identifying individual proteins of a certain kind in volume images, is presented. Grey-level and gradient magnitude information is combined in the watershed algorithm to extract stable borders. These are used in a subsequent hierarchical matching algorithm. The matching algorithm uses a distance transform to search for local best fits between the edges of a template and edges in the underlying image. It is embedded in a resolution pyramid to decrease the risk of getting stuck in false local minima. This method makes it possible to find proteins attached to other proteins, or proteins appearing as split into parts in the images. It also decreases the amount of human interaction needed for identifying individual proteins of the searched kind. The method is demonstrated on a set of three volume images of the antibody IgG in solution.
\end{abstract}

\section{Introduction}

The study of the structure of proteins or protein complexes is often the key to understanding how flexible a protein is and how it can interact with, or bind to, other proteins or substances, see, for example [1,2. This, in turn, is important for understanding complicated biological systems, e.g., viral infections or the effects of a potential drug candidate. However, imaging of a protein or a protein complex is a difficult task. Atomic resolution (ngstrm scale) can be achieved through X-ray crystallography or nuclear magnetic resonance (NMR). Both have the drawbacks of being very time consuming and restricted to certain types of proteins. Using electron microscopy, almost atomic level is possible to achieve by making a $3 \mathrm{D}$ reconstruction based on averaging thousands of proteins of the same kind.

Here, images produced by an individual particle imaging method called Sidec $^{T M}$ Electron Tomography (SET), are studied. SET allows imaging of proteins in solution or in tissue at the $\mathrm{nm}$ scale. This is enough to reveal the main structural features of many proteins, how flexible they are, and how they interact with other proteins [3, 4. SET is also very fast in comparison with the other methods. 

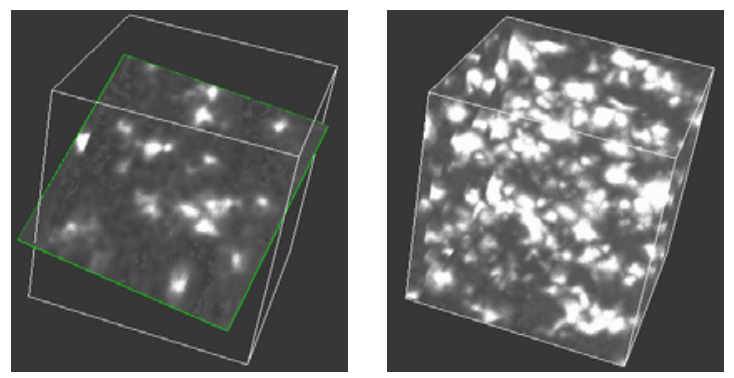

Fig. 1. A cross section (left), and a maximum intensity projection (right), of a SET volume image of a solution containing IgG antibody molecules. An IgG molecule is seen as the triangle-shaped object consisting of three parts, almost in the middle of the cross section

Briefly described, the SET images are generated as follows. 2D projections of the flash frozen sample, in our case a solution, are collected at several tiltangles in the transmission electron microscope. These projections are used to reconstruct the 3D sample by filtered back projection and a refinement method denoted Constrained Maximum Entropy Tomography, COMET [5]. A cross section of a SET volume image of a protein solution containing antibody molecules is shown in Figure 1 (left), and a maximum intensity projection in the direction of the cutting plane (right).

Each SET volume often contain hundreds of objects of which only one or a few are of interest. The proteins have very small volumes, each consisting of only a few thousand voxels, and the shape information is, hence, limited. The proteins of interest are so far found by visual inspection of objects having approximately the correct size after thresholding the intensity. The visual inspection is very time-consuming and the reconstructions also suffer from noise and varying background.

In this paper a segmentation method is presented which reduces the need for human interaction. The method also solves, partly or completely, the problems of varying background, objects touching other objects, and objects appearing as split into parts in the images. These problems can not be solved efficiently by grey-level thresholding in combination with size discrimination. The objects of interest will have different sizes depending on the background, and the size limit therefore needs to be generous not to discard true objects. This in turn increases the amount of objects to be visually judged. Objects of interest touching other objects will have a too large volume to pass the size discrimination step and they will, hence, easily be left unidentified. Objects that have been split into more than one connected component by the threshold will each have a too small volume to pass the size discrimination step and will also easily be left unidentified. The method presented here combines intensity, edge, and shape information using the watershed algorithm [6, 7] and the hierarchical chamfer matching algorithm [8] in a method which handles the problems of varying background, touching or split objects, and need for large amount of human interaction. 


\section{Method}

The core of the segmentation method presented here is the chamfer matching algorithm. It was originally presented in [9], and embedded in a resolution pyramid in $[8$, therefore the name hierarchical chamfer matching (HCMA). For all template matching algorithms a template is needed, and the image to be searched should represent the same type of information as the template. In the chamfer matching algorithm the template is a binary representation of the shape through, e.g., the contours of a typical object of interest and the images to be searched should therefore contain contour information. The chamfer matching method then finds good fits between the template contour and contours in the image by searching for local minima of a matching function. The matching function is an approximation of the $L^{2}$ norm between the template and the contour image. A resolution pyramid with $l$ levels of the contour image, where the resolution is lowered at each higher level of the pyramid, is calculated. Chamfer matching is then performed at the top (level $l$ ) of the pyramid, that is in the image with lowest resolution. Good match positions are transferred to the next, lower, level of the pyramid where they serve as starting positions for another round of matching. This continues until matching has been performed in the original image, i.e., the base of the pyramid. When no a priori information is available about the possible local transformations of objects in the image, this multi-resolution approach is necessary for template matching to be a feasible segmentation method.

To extract the contours in the image a seeded watershed (WS) algorithm run on gradient magnitude information, similar to the first steps of the cell nuclei segmentation method in [10, is used. Gradient magnitude information reveals local intensity changes in an image. At places where the local contrast is high, e.g., on the border between a bright object and dark background, the gradient magnitude will be high, while at places with low local contrast, e.g., in the background or in the interior of objects, the gradient magnitude will be low. WS segmentation [6] is a region growing method in which regions grow from all local minima in an image based on grey-level information until they meet other regions. The algorithm is easily understood by interpreting the intensities in an image as a topological map. If a hole is drilled in each local hollow before the map is submerged in water, water will start to fill the map from the hollows. Where two different water basins are about to meet, a dam, or watershed is built to prevent water originating from different hollows to blend. When the entire map has been submerged in water, the resulting regionalization, or the separating watersheds, constitute the segmentation. The algorithm can be applied to different types of information and it has been used in a variety of situations, see e.g., 7, 11,12, for overviews. Instead of growing regions from every local minimum seeds can be placed in the image as markers of regions of interest, see e.g., 7, 12. The WS algorithm is then run as described above with the exception that watersheds are only built between regions that each contain a seed.

In the rest of this section we will explain how a template is constructed (or found), how the binary borders are extracted by using a seeded WS algorithm, 

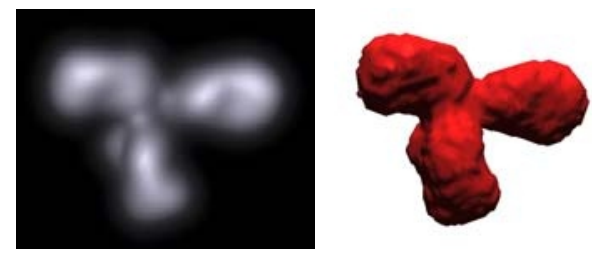

Fig. 2. A volume rendering of the density reconstruction of an antibody, PDB identification 1igt (left), and a surface rendering of the thresholded volume (right)

and finally how the HCMA algorithm is used to identify objects corresponding to a certain shape in a volume image.

\subsection{Template Construction}

Two types of templates can be discussed for the application in consideration here. Either a typical protein molecule of interest can be found by visual inspection in a SET volume and extracted, or, better, if the atomic structure of the protein is solved and entered into the protein data bank [13, this information can be used to create a template. The latter approach was used in the examples presented here. A volume image where grey-levels depict density in a similar manner as in a SET volume can be constructed by substituting the atomic positions by a gauss kernel weighted by the size of the atom [14, see Figure 2 for such a volume image. Note that the shape of a protein in solution is neither fixed nor always the same, as parts of a protein are more or less flexible. The shape of the template and objects of interest in the image will, hence, not be exactly the same. Once an image of a protein has been constructed, the binary edges to be used as a template need to be extracted. This is done by grey-level thresholding and keeping all voxels of the template that has a face neighbor in the background. The template voxels are described as points in a coordinate system with the origin in the center of the template. This way of storing the template enables simple scaling and rotation of the template in the matching procedure by multiplication with a constant and rotation matrices, respectively.

\subsection{Binary Contour Extraction}

As mentioned in the Introduction, the background varies and a uniform greylevel threshold does not give a satisfactory regionalisation. Instead, a seeded WS algorithm applied to gradient magnitude information is used to achieve a regionalisation stable for smooth background changes. Borders to be used in the subsequent matching are extracted as voxels in the object region having a face neighbor in the background. The different steps to extract the borders are illustrated on part of a 2D slice from a SET volume in Figure 3 .

Seeds corresponding to object regions and the background region should be placed in the image. The SET volumes contain bright objects on a dark background. Object seeds should, hence, be placed in bright regions of the image, 


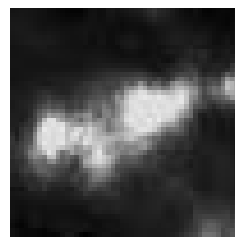

(a)

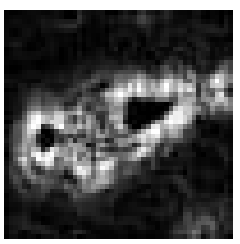

(b)

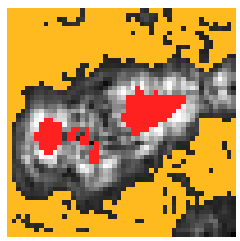

(c)

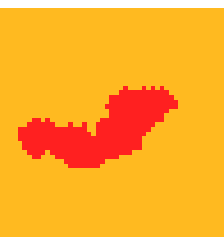

(d)

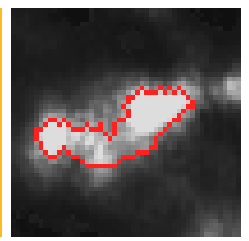

(e)

Fig. 3. The steps to extract the borders illustrated on small part of a $2 \mathrm{D}$ slice from one of the SET volumes. The original intensity image (a), the gradient magnitude image (b), and the background and object seeds (c). The watershed regionalization(d), and the extracted borders of the object region overlayed on the intensity image

and the background seed should be placed in the dark region of the image. Object seeds are found by thresholding the intensity image at a high threshold, $T O$, known to segment regions definitely inside objects. All voxels above this threshold are given a label stating that they belong to the object region. The background intensity varies to much to use an intensity threshold for background seeding. However, these variations have low frequency and therefore the gradient magnitude image, calculated as described below, is used to find a good background seed. In the gradient magnitude image, all values below a low threshold, $T B$, corresponding to flat regions in the original intensity image, are given a label stating that they are possibly part of the background seed. Of these, only the largest connected component is kept as the single background seed. Since most of the SET volumes are background the background seed will cover most of the volume, see Figure 3 (c).

Gradient magnitude information is a measure of local contrast in an image. A gradient magnitude image can be calculated by filtering the image with one, or a set of, local contrast detecting filters, see e.g. [15] for a number of commonly used filters. A set of six 3D Sobel filters are used here, each detecting edges in a different direction. The filter responses in each position of the image are combined to produce the gradient magnitude of that position. In Figure 3 (b), a gradient magnitude image calculated using a set of 2D Sobel filters are shown.

The WS algorithm can be efficiently implemented using sorted pixels lists [16]. In the method described in [16, thick watersheds often result, as image elements located at equal distance from two regions become part of the watershed. In the implementation used here, the water flows around these elements and as a final step these elements are assigned to the region to which most if its neighbors belong. In our implementation, only regions, and no separating watersheds, are the result, as each element of the watersheds is assigned to one of the regions it separates. In a seeded WS algorithm regions start to grow from every local minimum in the image, but as soon as a region containing a seed meets an unseeded region, the unseeded region is conquered by the seeded region. After the WS segmentation, we will, hence, have two regions, object and background, covering the whole image, see Figure 3 (d). 


\subsection{Hierarchical Chamfer Matching}

The volumes are rather big and the proteins can have any position and rotation. The HCMA generally needs a starting point close to the optimal position. Very many starting positions are therefore needed in order to guarantee finding the best matches. The matching is performed in a resolution pyramid to speed up the matching and to avoid getting caught in false local minima. The matching is started at low resolution, and positions with reasonably good match scores are kept to the next level. This means that at the best resolution level, that is the original contour image, only rather good positions need to be explored.

From the original contour image, a resolution pyramid is constructed as follows. Each $2 \times 2 \times 2$ block of voxel at one resolution level is represented by a single voxel at the next higher level. This means that the size of the image is reduced by $7 / 8$ at each level. The lowest value in the $2 \times 2 \times 2$ block is transferred to the corresponding position at the higher level. In the case where the starting image is a binary edge image, this corresponds to an OR-pyramid, since if any of the 8 lower level voxels is a zero, the corresponding voxel at the level in consideration becomes a zero.

A weighted distance transform (DT) is calculated from the contours at all levels of the pyramid. In the resulting distance images all voxels are thus given a value corresponding to the distance to the closest contour voxel. Local distance weights are propagated over the image in two passes [17. This propagation of local distances is known as chamfering, and weighted DTs are therefore sometimes called chamfer DTs, hence also the name of the matching procedure. The weights, or local distances, used are 3,4,5 for the face, edge, and vertex neighbors respectively. These weights are low and produces a DT that is rotation independent enough for matching purposes [18. During the first pass the image is processed from left to right, top to bottom and front to back. During the second pass the image is processed from right to left, bottom to top and back to front. The voxel under consideration is given the minimum value of itself and the values of its already visited neighbors, each increased by their respective local distance.

The matching is started at the top (level $l$ ) of the distance transformed pyramid. The template is transformed to the corresponding resolution, placed over the distance image and rotated according to the starting positions, found as described below. The similarity is calculated as the sum of the square of all distance values hit by the template. This means that the lower the sum is, the better is the match. Using the sum of each value hit by the template squared, instead of just the sum, is preferable as it introduces fewer false minima [19. A steepest descent and a line search method is used to find the closest local optimum for each starting position [20. Iteratively, a search step is taken in the steepest descent direction if it produces a smaller similarity measure. If not, the step length is halved until it does, or until the step length is so small that the local minimum can be considered reached. For each step, the steepest descent direction is calculated from all seven parameters (three translational, three rotational, and scale). The gradient for each parameter is approximated by centered 
difference, where a translational step is the side length of a voxel, a rotational step is the angle which makes a point on the edge of the template move one voxel side in length, and a scale step is a $2 \%$ change in scale.

From one level to the next, only the scores better than a threshold, $T 1$, depending on the size of the template and the resolution level, are kept. Once the matching is done at all levels, the positions that are left are presented according to their similarity measures in ascending order, that is, the position with the lowest score is presented first. This means that visual inspection is needed only for judging positions where the image strongly resembles the template. When a few false objects have been presented, the visual inspection can be stopped as the remaining positions are unlikely to correspond to true objects of interest. Since there are many objects in the volumes but rather few true molecules of interest we believe this final decision should not be replaced by a fixed threshold, but should be performed through visual inspection by human experts.

Starting positions, each consisting of a center location and a rotation for the template, could be distributed evenly throughout the highest level $(l)$ of the resolution pyramid. This would result in a very large amount of starting positions. In addition, many of these starting positions would be located in the background, hence, requiring more search steps to reach a local minimum. The local minima reached from locations in the background are also more likely to correspond to false hits, as the contours contributing to the local minima can consist of contours from two or more different objects. To reduce the number of starting positions and at the same time increase the probability for a starting position to lead to a true hit, starting locations should only be chosen in the interior of the object region resulting from the WS segmentation. This is achieved by calculating a resolution pyramid of the object region from the WS segmentation, with as many levels as in the contour image pyramid. It is not beneficial to chose starting locations too far into object regions, since many search steps then will be needed to reach a good local minimum. A DT was calculated from the background into the object region, and starting locations were only chosen at distances corresponding to the distance between the center and the closest contour point of the template at that resolution. Once a starting location has been chosen, no more starting locations are picked closer to the location than half the size of the template. For each of these starting locations, rotations were generated with an angular spacing of $\pi$ in each direction of rotation, leading to a total number of 4 starting positions for each location. Scaling of the template was not considered for generating starting positions, as the approximate scale is known. However, scale is allowed to change during matching.

\section{$3 \quad$ Results}

The method was tested on three SET volume images of solutions containing antibody IgG, each with one visually identified IgG molecule. The volumes were of size $150 \times 150 \times 150$ voxels, with a voxel size of $5.24 \times 5.24 \times 5.24$, and greyvalues in the interval [0,255]. A template with a resolution of $2 \mathrm{~nm}$ and a voxel 

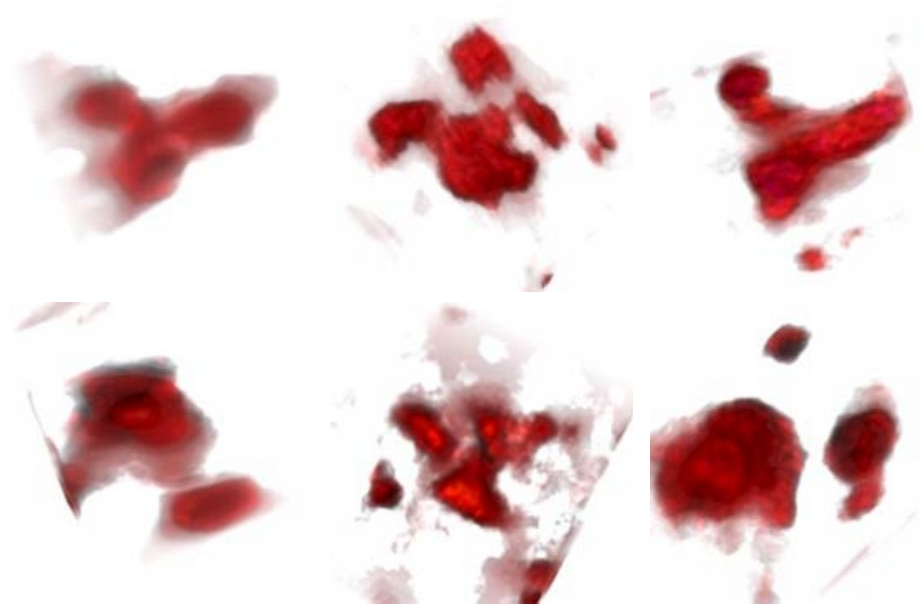

Fig. 4. Volume renderings of the sub-volumes corresponding to the the best (top row) and second best (bottom row) for each of the three volumes. The visually judged true IgG proteins are at the best position for the first and third volume and at the second best position in the second volume

size of $5.24 \times 5.24 \times 5.24$ was constructed from PDB (PDB identification 1igt). The constructed image was thresholded at grey-level 50 to extract the template borders. The template borders consisted of 1225 voxels, all fitting inside a box of size $22 \times 30 \times 27$ voxels. In Figure 2, a volume rendering of the constructed intensity image and a surface rendering of the thresholded volume are shown. The HCMA was run using $l=3$, that is, a resolution pyramid with three levels. The threshold $T O$, used to identify object seeds in the original intensity image, was 230 , and the threshold $T B$, used to identify the background seed in the gradient magnitude image, was 25 . These thresholds can be easily chosen by studying a small part of the volume. They are not critical, as a range of values will serve the purpose. The rejection threshold $T 1$ was (the number of elements in the template $\times 10) /($ hierarchical level), which only rejected very bad positions.

Volume renderings of sub-volumes of the original image at the two best positions in each image are shown in Figure 4. The three visually identified IgG molecules were in the first and third images found at the positions that best matched the template, and in the second image it was found at the second best position. Note that the structure corresponding to the best position in the second volume shows strong resemblance to an IgG protein and it might very well be a true IgG protein not found by the segmentation algorithm formerly used. The structures at the second best positions in the first and third volume, do not correspond to true IgG proteins. In these two cases the borders from different objects have contributed to their match with the template. Some such cases can, most likely, be identified and removed by studying the mean intensity inside the template placed at the position. We hope to test the method on more volumes in the near future, when more volumes will become available. 


\section{Discussion}

As shown in the result section, the proposed method manages to find the objects of interest despite the problems of varying background and objects appearing as connected or split, and despite that the shape of the proteins in solution differ considerably from the crystal structure used as the template. The amount of visual inspection needed to find specific proteins can, hence, be reduced. In situations when the structure of a searched protein is not deposited in PDB, a protein to serve as a template must be found by visual inspection of the reconstructed volumes. Already identified proteins of interest can also be used to re-search volumes and possibly find proteins of interest whose shape differ too much from the crystal shape to be found in a first match procedure.

This paper shows that the proposed method is a promising tool in the analysis of SET images. The strength of the approach is that several types of information and a robust matching measure are combined to produce a stable identification method. In the object seeding process, information that true objects of interest internally have very high intensities is incorporated. Another possible object seeding approach would be to mark local maxima higher than a certain height as object seeds, as in [10. This would remove the choice of the object threshold, but it would instead lead to many more object regions originating from local maxima that not corresponding to true proteins, as their internal intensity is to low. The problem with varying background is handled by finding the background seed in the gradient magnitude image and using a seeded WS algorithm to extract binary borders. Finally, shape information is used to search for positions in the image where the extracted borders resemble the shape of the template.

The method, or parts of it, can likely be improved by using other, more sophisticated, edge detecting algorithms and optimization methods. However, that the method works despite the simple edge detection and optimization algorithms, demonstrates its robustness. To be able to fine-tune the algorithms and parameters included in the method, its performance on more SET volumes of proteins of several different types need to be investigated.

\section{References}

1. Norel, R., Petrey, D., Wolfson, H.J., Nussinov, R.: Examination of shape complementarity in docking of unbound proteins. Prot. Struct. Func. Gen. 36 (1999) 307-317

2. Liang, J., Edelsbrunner, H., Woodward, C.: Anatomy of protein pockets and cavities: Measurement of binding site geometry and implications for ligand design. Prot. Sci. 7 (1998) 1884-1897

3. Banyay, M., Gilstring, F., Hauzenberger, E., Öfverstedt, L.G., Eriksson, A.B., Krupp, J.J., Larsson, O.: Three-dimensional imaging of in situ specimens with low-dose electron tomography to analyze protein conformation. Assay Drug Dev. Technol. 2 (2004) 561-567

4. Sandin, S., Öfverstedt, L.G., Wikström, A.C., Wrange, O., Skoglund, U.: Structure and flexibility of individual immunoglobulin $\mathrm{G}$ molecules in solution. Structure 12 (2004) 409-415 
5. Skoglund, U., Öfverstedt, L.G., Burnett, R., Bricogne, G.: Maximum-entropy three-dimensional reconstruction with deconvolution of the contrast transfer function: A test application with adenovirus. J. Struct. Biol. 117 (1996) 173-188

6. Beucher, S., Lantuéjoul, C.: Use of watersheds in contour detection. In: International Workshop on Image Processing: Real-time and Motion Detection/ Estimation. (1979) 2.1-2.12

7. Meyer, F., Beucher, S.: Morphological segmentation. J. Vis. Comm. Im. Repr. 1 (1990) 21-46

8. Borgefors, G.: Hierarchical chamfer matching: A parametric edge matching algorithm. IEEE Trans. Pat. Anal. Mach. Intell. 10 (1988) 849-865

9. Barrow, H.G., Tenenbaum, J.M., Bolles, R., Wolf, H.C.: Parametric correspondence and chamfer matching: Two new techniques for image matching. In: Proc. 5th Int. Joint Conf. Artif. Intell., Cambridge, Massachusetts (1977) 659-663

10. Wählby, C., Sintorn, I.M., Erlandsson, F., Borgefors, G., Bengtsson, E.: Combining intensity, edge, and shape information for $2 \mathrm{D}$ and $3 \mathrm{D}$ segmentation of cell nuclei in tissue sections. J. Micr. 215 (2004) 67-76

11. Vincent, L.: Morphological grayscale reconstruction in image analysis: Applications and efficient algorithms. IEEE Trans. Im. Proc. 2 (1993) 176-201

12. Beucher, S.: The watershed transformation applied to image segmentation. Scanning Microscopy 6 (1992) 299-314

13. Berman, H., Westbrook, J., Feng, Z., Gilliland, G., Bhat, T., Weissig, H., Shindyalova, I., Bourne, P.: The protein data bank. Nucl. Ac. Res. 28 (2000) 235242

14. Pittet, J.J., Henn, C., Engel, A., Heymann, J.B.: Visualizing 3D data obtained from microscopy on the internet. J. Struct. Biol. 125 (1999) 123-132

15. Sonka, M., Hlavac, V., Boyle, R.: 4. In: Image Processing, Analysis, and Machine Vision. 2nd edn. Brooks/Cole Publishing Company (1999) 77-88

16. Vincent, L., Soille, P.: Watersheds in digital spaces: An efficient algorithm based on immersion simulations. IEEE Trans. Pat. Anal. Mach. Intell. 13 (1991) 583-597

17. Rosenfeld, A., Pfaltz, J.L.: Sequential operations in digital picture processing. J. Ass. Comp. Mach. 13 (1966) 471-494

18. Borgefors, G.: On digital distance transforms in three dimensions. Comp. Vis. Im. Underst. 64 (1996) 368-376

19. Borgefors, G.: An improved version of the chamfer matching algorithm. In: 7th Int. Conf. on Pat. Rec., Montreal, Canada (1984) 1175-1177

20. Nash, S.G., Sofer, A.: 10.5, 11.1. In: Linear and Nonlinear Programming. McGrawHill (1996) 